\title{
AN OPERATOR ASSOCIATED WITH DE BRANGES SPACES AND UNIVERSALITY LIMITS
}

\author{
D. S. LUBINSKY
}

ABSTRACT. Under suitable conditions on a measure, universality limits $f(\cdot, \cdot)$ that arise in the bulk, unitary case, are reproducing kernels of de Branges spaces of entire functions. In the classical case, $f$ is the sinc kernel

$$
f(s, t)=\frac{\sin \pi(s-t)}{\pi(s-t)},
$$

but other kernels can arise. We study the linear operator

$$
\mathcal{L}[h](x)=\int_{-\infty}^{\infty} f(s, x) h(s) d s,
$$

establishing inequalities, and deducing some conditions for $f$ to equal the sinc kernel.

\section{IntRoduCtion AND RESUlTS ${ }^{1}$}

Let $\mu$ be a finite positive Borel measure on $\mathbb{R}$ with all moments $\int x^{j} d \mu(x)$, $j \geq 0$, finite, and with infinitely many points in its support. Then we may define orthonormal polynomials

$$
p_{n}(x)=\gamma_{n} x^{n}+\ldots, \gamma_{n}>0,
$$

$n=0,1,2, \ldots$ satisfying the orthonormality conditions

$$
\int p_{n} p_{m} d \mu=\delta_{m n}
$$

Throughout we use $\mu^{\prime}(x)=\frac{d \mu}{d x}$ to denote the almost everywhere existing Radon-Nikodym derivative of $\mu$.

Orthogonal polynomials play an important role in random matrix theory, especially in the unitary case [2], [4], [17]. One of the key limits there involves the reproducing kernel

$$
K_{n}(x, y)=\sum_{k=0}^{n-1} p_{k}(x) p_{k}(y) .
$$

Because of the Christoffel-Darboux formula, it may also be expressed as

$$
K_{n}(x, y)=\frac{\gamma_{n-1}}{\gamma_{n}} \frac{p_{n}(x) p_{n-1}(y)-p_{n-1}(x) p_{n}(y)}{x-y}, x \neq y .
$$

Date: August 30, 2009.

${ }^{1}$ Research supported by NSF grant DMS0400446 and US-Israel BSF grant 2004353 
Define the normalized kernel

$$
\widetilde{K}_{n}(x, y)=\mu^{\prime}(x)^{1 / 2} \mu^{\prime}(y)^{1 / 2} K_{n}(x, y) .
$$

The simplest case of the universality law is the limit

$$
\lim _{n \rightarrow \infty} \frac{\widetilde{K}_{n}\left(\xi+\frac{a}{\widetilde{K}_{n}(\xi, \xi)}, \xi+\frac{b}{\widetilde{K}_{n}(\xi, \xi)}\right)}{\widetilde{K}_{n}(\xi, \xi)}=\frac{\sin \pi(a-b)}{\pi(a-b)},
$$

involving the sinc kernel. It describes the distribution of spacing of eigenvalues of random matrices. Typically this limit holds uniformly for $\xi$ in the interior of the support of $\mu$ and $a, b$ in compact subsets of the real line. See [1], [2], [4], [5], [6], [8], [9], [10], [11], [12], [15], [16], [20], [21], [22], [24]. Of course, when $a=b$, we interpret $\frac{\sin \pi(a-b)}{\pi(a-b)}$ as 1 .

One reason for the appearance of the sinc kernel $\frac{\sin \pi(a-b)}{\pi(a-b)}$ in (1.4) is that it is the reproducing kernel for the classical Paley-Wiener space $P W_{\pi}$. Recall that for $\sigma>0, P W_{\sigma}$ consists of entire functions of exponential type $\leq \sigma$ that are square integrable along the real axis, with the usual $L_{2}$ norm. In the course of investigating (1.4), the author found that other reproducing kernels can arise - namely reproducing kernels of de Branges spaces. Under mild conditions on the measure, the de Branges spaces that were obtained equal classical Paley-Wiener spaces as sets. It is the purpose of this paper, to further investigate this relationship.

de Branges spaces [3, p. 50], [14, p. 983. ff], [19, p. $793 \mathrm{ff}$.$] are built$ around the Hermite-Biehler class. An entire function $E$ is said to belong to the Hermite-Biehler class if it has no zeros in the upper half-plane $\mathbb{C}^{+}=$ $\{z: \operatorname{Im} z>0\}$ and

$$
|E(z)| \geq|E(\bar{z})| \text { for } z \in \mathbb{C}^{+} .
$$

We write $E \in \overline{H B}$. Recall that the Hardy space $H^{2}\left(\mathbb{C}^{+}\right)$is the set of all functions $g$ analytic in the upper-half plane, for which

$$
\sup _{y>0} \int_{-\infty}^{\infty}|g(x+i y)|^{2} d x<\infty
$$

Given an entire function $g$, we let

$$
g^{*}(z)=\overline{g(\bar{z})} .
$$

\section{Definition 1.1}

The de Branges space $\mathcal{H}(E)$ corresponding to the entire function $E \in \overline{H B}$, is the set of all entire functions $g$ such that both $g / E$ and $g^{*} / E$ belong to $H^{2}\left(\mathbb{C}^{+}\right)$, with

$$
\|g\|_{E}=\left(\int_{-\infty}^{\infty}\left|\frac{g}{E}\right|^{2}\right)^{1 / 2}<\infty
$$


$\mathcal{H}(E)$ is a Hilbert space with inner product

$$
(g, h)=\int_{-\infty}^{\infty} \frac{g \bar{h}}{|E|^{2}}
$$

Remarkably, one may construct an explicit reproducing kernel for $\mathcal{H}(E)$ from $E$ [14, p. 984], [19, p. 793]. Indeed, if we let

$$
\mathcal{K}(\zeta, z)=\frac{i}{2 \pi} \frac{E(z) \overline{E(\zeta)}-E^{*}(z) \overline{E^{*}(\zeta)}}{z-\bar{\zeta}},
$$

then for all $\zeta, \mathcal{K}(\zeta, \cdot) \in \mathcal{H}(E)$ and for all complex $\zeta$ and all $g \in \mathcal{H}(E)$,

$$
g(\zeta)=\int_{-\infty}^{\infty} \frac{g(t) \overline{\mathcal{K}(\zeta, t)}}{|E(t)|^{2}} d t
$$

The classical de Branges spaces are the Paley-Wiener spaces $P W_{\sigma}$. There one may take $E(z)=\exp (-i \sigma z)$, and the norm is just

$$
\|g\|_{L_{2}(\mathbb{R})}=\left(\int_{-\infty}^{\infty}|g|^{2}\right)^{1 / 2}
$$

while

$$
\mathcal{K}(\zeta, z)=\frac{\sin \sigma(z-\bar{\zeta})}{\pi(z-\bar{\zeta})}
$$

We write

$$
\mathcal{H}(E)=P W_{\sigma}
$$

if the two spaces are equal as sets, and have equivalent norms (we do not imply isometric isomorphism). Recall that having equivalent norms means that for some $C>1$ independent of $g \in P W_{\sigma}$,

$$
C^{-1}\|g\|_{L_{2}(\mathbb{R})} \leq\|g\|_{E} \leq C\|g\|_{L_{2}(\mathbb{R})} .
$$

The closed graph theorem can be used to show that this norm equivalence follows from mere equality as sets.

The main conclusion of our recent paper [12] was that universality limits in the bulk are reproducing kernels of de Branges spaces that equal classical Paley-Wiener spaces. Moreover, any such reproducing kernel can arise as a universality limit. The first explicit example of a kernel other than the sinc kernel in this setting, has been given by Moreno, Finkelshtein and Sousa [18]. They considered absolutely continuous measures with $\mu^{\prime}$ having a jump discontinuity, and used the Riemann-Hilbert method to give a precise and beautiful description of "universality at the jump".

By a universality limit, we mean a limit of some subsequence of $\left\{f_{n}\right\}$, where

$$
f_{n}(a, b)=\frac{K_{n}\left(\xi+\frac{a}{\widetilde{K}_{n}(\xi, \xi)}, \xi+\frac{b}{\widetilde{K}_{n}(\xi, \xi)}\right)}{K_{n}(\xi, \xi)} .
$$

More precisely, we showed: 


\section{Theorem 1.2}

Let $\mu$ be a measure with compact support. Assume that $\mu$ is absolutely continuous in a neighborhood of $\xi$, and in that neighborhood, for some $C>1$,

$$
C^{-1} \leq \mu^{\prime} \leq C
$$

Define $\left\{f_{n}\right\}$ by (1.9).

(a) $\left\{f_{n}(\cdot, \cdot)\right\}$ is a normal family in compact subsets of $\mathbb{C}^{2}$.

(b) Let $f(\cdot, \cdot)$ be the limit of some subsequence $\left\{f_{n}(\cdot, \cdot)\right\}_{n \in \mathcal{S}}$. Then $f$ is an entire function of two variables, that is real valued in $\mathbb{R}^{2}$ and has $f(0,0)=1$. Moreover, for some $\sigma>0, f(\cdot, \cdot)$ is entire of exponential type $\sigma$ in each variable.

(c) Define

$$
L(u, v)=(u-v) f(u, v), u, v \in \mathbb{C} .
$$

Let $a \in \mathbb{C}$ have $\operatorname{Im} a>0$ and let

$$
E_{a}(z)=\sqrt{2 \pi} \frac{L(\bar{a}, z)}{|L(a, \bar{a})|^{1 / 2}} .
$$

Then $f$ is a reproducing kernel for $\mathcal{H}\left(E_{a}\right)$. In particular, for all $z, \zeta$,

$$
f(z, \bar{\zeta})=\frac{i}{2 \pi} \frac{E_{a}(z) \overline{E_{a}(\zeta)}-E_{a}^{*}(z) \overline{E_{a}^{*}(\zeta)}}{z-\bar{\zeta}} .
$$

(d) Moreover,

$$
\mathcal{H}\left(E_{a}\right)=P W_{\sigma}
$$

and the norms $\|\cdot\|_{E_{a}}$ of $\mathcal{H}\left(E_{a}\right)$ and $\|\cdot\|_{L_{2}(\mathbb{R})}$ of $P W_{\sigma}$ are equivalent.

In that same paper, we showed that for sequences of measures, any such reproducing kernel can arise as a universality limit. However, we could not show this for a fixed measure. That remains an interesting unsolved problem.

It is the aim of this paper, to further explore the properties of the de Branges spaces above. We shall especially be interested in the case when $\xi$ is a Lebesgue point of $\mu^{\prime}$, so that

$$
\lim _{h \rightarrow 0+} \frac{1}{2 h} \int_{\xi-h}^{\xi+h}\left|\mu^{\prime}(t)-\mu^{\prime}(\xi)\right| d t=0 .
$$

Define the operator

$$
\mathcal{L}[h](x)=\int_{-\infty}^{\infty} h(t) f(t, x) d t
$$

for $h \in L_{2}(\mathbb{R})$, and with $f$ as in Theorem 1.2. This is well defined as $f(\cdot, x) \in L_{2}(\mathbb{R})$. Note that if $f$ was the sinc reproducing kernel for $P W_{\sigma}$, that is

$$
f(x, s)=\frac{\sin \sigma(x-s)}{\pi(x-s)}
$$


then for all $h \in P W_{\sigma}$, and all real $x$ [23, Cor. 1.10.5, p. 95]

$$
\mathcal{L}[h](x)=h(x) .
$$

Conversely, this relation establishes that $f$ is the reproducing kernel for $P W_{\sigma}$, and hence is the sinc kernel by uniqueness of reproducing kernels. Thus one might hope to investigate the distance from $f$ to the sinc kernel by studying the operator $\mathcal{L}$.

We shall prove:

\section{Theorem 1.3}

Assume the hypotheses of Theorem 1.2 and in addition that the Lebesgue point condition (1.15) holds. Let $\operatorname{Im} \alpha>0$. For all $h \in L_{2}(\mathbb{R})$,

$$
\int_{-\infty}^{\infty} \mathcal{L}[h]^{2} \leq \int_{-\infty}^{\infty} h^{2}
$$

$$
\int_{-\infty}^{\infty} \frac{\mathcal{L}[h]^{2}}{\left|E_{\alpha}\right|^{2}} \leq \int_{-\infty}^{\infty} h^{2}
$$

$$
\int_{-\infty}^{\infty} \frac{\mathcal{L}\left[h /\left|E_{\alpha}\right|^{2}\right]^{2}}{\left|E_{\alpha}\right|^{2}} \leq \int_{-\infty}^{\infty} \frac{h^{2}}{\left|E_{\alpha}\right|^{2}}
$$

$$
\int_{-\infty}^{\infty} \mathcal{L}\left[h /\left|E_{\alpha}\right|^{2}\right]^{2} \leq \int_{-\infty}^{\infty} \frac{h^{2}}{\left|E_{\alpha}\right|^{2}}
$$

One corollary is

\section{Corollary 1.4}

Assume the hypotheses of Theorem 1.2 and in addition that the Lebesgue point condition (1.15) holds. Let $\operatorname{Im} \alpha>0$. Let $g \in P W_{\sigma}, \beta \in[0,2]$ and $\gamma \geq 2$. Then

$$
\int_{-\infty}^{\infty} \frac{|g|^{2}}{\left|E_{\alpha}\right|^{\beta}} \leq \int_{-\infty}^{\infty} \frac{|g|^{2}}{\left|E_{\alpha}\right|^{\gamma}} .
$$

In $\left[12\right.$, Theorem 5.3], we showed that $f(0, \cdot)$ has only real zeros $\left\{\rho_{j}\right\}_{j \neq 0}$, where

$$
\ldots<\rho_{-2}<\rho_{-1}<0=\rho_{0}<\rho_{1}<\rho_{2}<\ldots .
$$


It was also shown $[12$, Theorem $5.3,5.4]$ that $\left\{\frac{f\left(\rho_{j}, \cdot\right)}{\sqrt{f\left(\rho_{j}, \rho_{j}\right)}}\right\}_{j}$ is a complete orthonormal set in $\mathcal{H}\left(E_{\alpha}\right)$, so that

$$
\int_{-\infty}^{\infty} f\left(\rho_{j}, x\right) f\left(\rho_{k}, x\right) \frac{d x}{\left|E_{\alpha}(x)\right|^{2}}=\delta_{j k} f\left(\rho_{j}, \rho_{j}\right),
$$

and for any $g \in P W_{\sigma}=\mathcal{H}\left(E_{\alpha}\right)$, there is the convergent orthonormal expansion

$$
g(z)=\sum_{j=-\infty}^{\infty} g\left(\rho_{j}\right) \frac{f\left(\rho_{j}, z\right)}{f\left(\rho_{j}, \rho_{j}\right)} .
$$

In particular, this implies that

$$
\int_{-\infty}^{\infty} \frac{|g(x)|^{2}}{\left|E_{\alpha}(x)\right|^{2}} d x=\sum_{j=-\infty}^{\infty} \frac{\left|g\left(\rho_{j}\right)\right|^{2}}{f\left(\rho_{j}, \rho_{j}\right)}
$$

\section{Theorem 1.5}

Assume the hypotheses of Theorem 1.2 and in addition that the Lebesgue point condition (1.15) holds. Let $\operatorname{Im} \alpha>0$. For all $h \in L_{2}(\mathbb{R})$,

(a)

$$
\begin{aligned}
& \int_{-\infty}^{\infty}\left(h-\mathcal{L}\left[\frac{h}{\left|E_{a}\right|^{2}}\right]\right)^{2} \frac{1}{\left|E_{a}\right|^{2}} \\
\leq & \inf _{g \in P W_{\sigma}} \int_{-\infty}^{\infty}|h-g|^{2} \frac{1}{\left|E_{a}\right|^{2}} .
\end{aligned}
$$

(b)

$$
\begin{aligned}
& \int_{-\infty}^{\infty}(h-\mathcal{L}[h])^{2} \\
\leq & \inf _{g \in P W_{\sigma}}\left\{\int_{-\infty}^{\infty}|h-g|^{2}+\int_{-\infty}^{\infty}|g|^{2}\left(\frac{1}{\left|E_{a}\right|^{2}}-1\right)\right\} \\
\leq & \int_{-\infty}^{\infty} h^{2}-\sum_{j=-\infty}^{\infty} \frac{\mathcal{L}[h]^{2}\left(\rho_{j}\right)}{f\left(\rho_{j}, \rho_{j}\right)} .
\end{aligned}
$$

\section{Corollary 1.6}

Assume the hypotheses of Theorem 1.2 and in addition that the Lebesgue point condition (1.15) holds. Let $\operatorname{Im} \alpha>0$. Let $g \in P W_{\sigma}$. Then the following are equivalent:

$$
\mathcal{L}[g](x)=g(x) \text { for all } x
$$




$$
\mathcal{L}[g]\left(\rho_{j}\right)=g\left(\rho_{j}\right) \text { for all } j
$$

$$
\int_{-\infty}^{\infty} \frac{|g|^{2}}{\left|E_{\alpha}\right|^{2}}=\int_{-\infty}^{\infty}|g|^{2} .
$$

Moreover, $f$ is the sinc kernel, that is for all $s, x$,

$$
f(s, x)=\frac{\sin \sigma(s-x)}{\pi(s-x)},
$$

iff any of (I), (II), (III) hold for all $g \in P W_{\sigma}$.

Part (III) above says that we have universality with the sinc kernel iff the spaces $P W_{\sigma}$ and $H\left(E_{\alpha}\right)$ are isometrically isomorphic. However, (1.29) does not necessarily imply that $\left|E_{\alpha}\right|=1$ in $\mathbb{R}$ [3]. Finally, we note that in [12], we considered also sequences of measures, and sequences of points rather than a fixed point $\xi$. The same extensions can be carried out, with very minor changes in the proofs, in the context of this paper.

\section{Proofs}

Our main tools are suitable scalings, Bessel's inequality, least squares, and the Geronimus type identity [12, Theorem 4.3(d), p. 385], [13, Theorem 2.1]

$$
\int_{-\infty}^{\infty} \frac{P(t)}{\left|E_{n, a}(t)\right|^{2}} d t=\int_{-\infty}^{\infty} P(t) d \mu(t),
$$

valid for all polynomials $P$ of degree $\leq 2 n-2$. Here $\operatorname{Im} a>0$, and

$$
E_{n, a}(t)=\sqrt{2 \pi} \frac{L_{n}(\bar{a}, t)}{\left|L_{n}(a, \bar{a})\right|^{1 / 2}},
$$

where

$$
\begin{aligned}
L_{n}(u, v) & =(u-v) K_{n}(u, v) \\
& =\frac{\gamma_{n-1}}{\gamma_{n}}\left(p_{n}(u) p_{n-1}(v)-p_{n-1}(u) p_{n}(v)\right)
\end{aligned}
$$

We let

$$
S_{n}[g](x)=\int K_{n}(x, t) g(t) d \mu(t)
$$

denote the $n$th partial sum of the orthonormal expansion with respect to the orthogonal polynomials for $\mu$, and $S_{n}^{*}$ that for $\frac{1}{\left|E_{n, a}\right|^{2}}$, so that

$$
S_{n}^{*}[g](x)=\int K_{n}(x, t) g(t) \frac{d t}{\left|E_{n, a}\right|^{2}} .
$$


Note that both have the same reproducing kernel $K_{n}$, as both share the same first $n-1$ orthogonal polynomials, recall (2.1). We assume that as $n \rightarrow \infty$ through the subsequence $\mathcal{S}$, that

$$
f_{n} \rightarrow f
$$

uniformly in compact sets. We shall make some elementary scalings and substitutions, and then take limits. We often use the abbreviation

$$
\kappa_{n}:=\tilde{K}_{n}(\xi, \xi)
$$

\section{Lemma 2.1}

Let $\operatorname{Im} \alpha>0$ and for $n \geq 1$,

$$
a=a(n)=\xi+\frac{\alpha}{\kappa_{n}} .
$$

(a) Then uniformly for $u$ in compact subsets of the plane,

$$
\lim _{n \in \mathcal{S}} E_{n, a}\left(\xi+\frac{u}{\kappa_{n}}\right) \mu^{\prime}(\xi)^{1 / 2}=E_{\alpha}(u)
$$

(b) Let $h \in L_{2}(\mathbb{R})$, let $r>0$, and

$$
g_{r}\left(\xi+\frac{s}{\kappa_{n}}\right)=\left\{\begin{array}{cc}
h(s), & s \in[-r, r] \\
0, & \text { otherwise }
\end{array} .\right.
$$

Then uniformly for $u$ in compact subsets of the plane,

$$
\begin{gathered}
\lim _{n \in \mathcal{S}} K_{n}(\xi, \xi) \int g_{r}^{2} d \mu=\int_{-r}^{r} h^{2}(s) d s \\
\lim _{n \in \mathcal{S}} K_{n}(\xi, \xi) \int g_{r}^{2} \frac{1}{\left|E_{n, a}\right|^{2}}=\int_{-r}^{r} \frac{h^{2}(s)}{\left|E_{\alpha}(s)\right|^{2}} d s \\
\lim _{n \in \mathcal{S}} S_{n}\left[g_{r}\right]\left(\xi+\frac{u}{\kappa_{n}}\right)=\int_{-r}^{r} h(s) f(u, s) d s \\
\lim _{n \in \mathcal{S}} S_{n}^{*}\left[g_{r}\right]\left(\xi+\frac{u}{\kappa_{n}}\right)=\int_{-r}^{r} h(s) f(u, s) \frac{d s}{\left|E_{\alpha}(s)\right|^{2}} .
\end{gathered}
$$




\section{Proof}

(a) Observe from (2.2) and (2.3) that

$$
\begin{aligned}
& E_{n, a}\left(\xi+\frac{u}{\kappa_{n}}\right) \mu^{\prime}(\xi)^{1 / 2} \\
= & \sqrt{2 \pi} \frac{(\bar{\alpha}-u) K_{n}\left(\xi+\frac{\bar{\alpha}}{\kappa_{n}}, \xi+\frac{u}{\kappa_{n}}\right)}{\kappa_{n}} \frac{1}{\left|(\alpha-\bar{\alpha}) \frac{K_{n}\left(\xi+\frac{\alpha}{\kappa_{n}}, \xi+\frac{\bar{\alpha}}{\kappa_{n}}\right)}{\kappa_{n}}\right|^{1 / 2}} \\
= & \sqrt{2 \pi} \frac{(\bar{\alpha}-u) f_{n}(\bar{\alpha}, u)}{\left|(\alpha-\bar{\alpha}) f_{n}(\alpha, \bar{\alpha})\right|^{1 / 2}} .
\end{aligned}
$$

Taking limits through $\mathcal{S}$, gives

$$
\begin{gathered}
\sqrt{2 \pi} \frac{(\bar{\alpha}-u) f(\bar{\alpha}, u)}{|(\alpha-\bar{\alpha}) f(\alpha, \bar{\alpha})|^{1 / 2}} \\
=\sqrt{2 \pi} \frac{L(\bar{\alpha}, u)}{|L(\alpha, \bar{\alpha})|^{1 / 2}}=E_{\alpha}(u),
\end{gathered}
$$

recall (1.11) and (1.12).

(b) We see that for large enough $n \in \mathcal{S}$,

$$
\begin{gathered}
\int g_{r}^{2} d \mu=\frac{1}{K_{n}(\xi, \xi)} \int_{-r}^{r} h^{2}(s) \frac{\mu^{\prime}\left(\xi+\frac{s}{\kappa_{n}}\right)}{\mu^{\prime}(\xi)} d s \\
\int g_{r}^{2} \frac{1}{\left|E_{n, a}\right|^{2}}=\frac{1}{K_{n}(\xi, \xi)} \int_{-r}^{r} h^{2}(s) \frac{d s}{\left|E_{n, a}\right|^{2}\left(\xi+\frac{s}{\kappa_{n}}\right) \mu^{\prime}(\xi)} .
\end{gathered}
$$

Then (2.9) follows using (1.15), while (2.10) follows more easily. Next,

$$
\begin{aligned}
S_{n}\left[g_{r}\right]\left(\xi+\frac{u}{\kappa_{n}}\right) & =\int_{-r}^{r} h(s) \frac{K_{n}\left(\xi+\frac{u}{\kappa_{n}}, \xi+\frac{s}{\kappa_{n}}\right)}{K_{n}(\xi, \xi)} \frac{\mu^{\prime}\left(\xi+\frac{s}{\kappa_{n}}\right)}{\mu^{\prime}(\xi)} d s \\
& =\int_{-r}^{r} h(s) f_{n}(u, s)\left\{1+\left(\frac{\mu^{\prime}\left(\xi+\frac{s}{\kappa_{n}}\right)}{\mu^{\prime}(\xi)}-1\right)\right\} d s .
\end{aligned}
$$

Here [11, proof of Lemma 5.2], [12, proof of Lemma 6.1] $f_{n}$ admits the bound

$$
\left|f_{n}(u, v)\right| \leq C_{1} e^{C_{2}(|\operatorname{Im} u|+|\operatorname{Im} v|)}
$$

where $C_{1}$ and $C_{2}$ do not depend on $n, u, v$, but for a given $R>0$, this holds for $|u|,|v| \leq R$ only when $n \geq n_{0}(R)$. Applying this bound (2.14), and the Lebesgue point condition (1.15), we see that the right-hand side in (2.13) has limit

$$
\int_{-r}^{r} h(s) f(u, s) d s
$$


as $n \rightarrow \infty$ through $\mathcal{S}$. So (2.11) follows. Similarly

$$
S_{n}^{*}\left[g_{r}\right]\left(\xi+\frac{u}{\tilde{K}_{n}}\right)=\int_{-r}^{r} h(s) f_{n}(u, s) \frac{1}{\mu^{\prime}(\xi)} \frac{d s}{\left|E_{n, a}\right|^{2}\left(\xi+\frac{s}{\kappa_{n}}\right)},
$$

and (2.12) follows.

We turn to the

\section{Proof of Theorem 1.3}

(I) Let $b>0$. We use Bessel's inequality in the form

$$
\int S_{n}\left[g_{r}\right]^{2} d \mu \leq \int g_{r}^{2} d \mu
$$

where $g_{r}$ chosen as in (2.8). Recall from (2.9) that

$$
\int g_{r}^{2} d \mu=\frac{1+o(1)}{K_{n}(\xi, \xi)} \int_{-r}^{r} h^{2}(s) d s
$$

while a substitution and (2.11) give

$$
\int S_{n}\left[g_{r}\right]^{2} d \mu \geq \frac{1}{K_{n}(\xi, \xi)} \int_{-b}^{b}\left|\int_{-r}^{r} h(s) f(t, s) d s+o(1)\right|^{2} \frac{\mu^{\prime}\left(\xi+\frac{t}{\kappa_{n}}\right)}{\mu^{\prime}(\xi)} d t .
$$

From this, (1.15), and the Bessel's inequality above, we obtain

$$
\int_{-b}^{b}\left|\int_{-r}^{r} h(s) f(t, s) d s\right|^{2} d t \leq \int_{-r}^{r} h^{2} .
$$

Now let $r \rightarrow \infty$ and then $b \rightarrow \infty$. The passage to the limit is justified, for example, by dominated convergence, since both $h(\cdot), f(t, \cdot) \in L_{2}(\mathbb{R})$.

(II) We use the Geronimus type formula (2.1), and Bessel's inequality:

$$
\int S_{n}\left[g_{r}\right]^{2} \frac{1}{\left|E_{n, a}\right|^{2}}=\int S_{n}\left[g_{r}\right]^{2} d \mu \leq \int g_{r}^{2} d \mu .
$$

Here from (2.11),

$$
\int S_{n}\left[g_{r}\right]^{2} \frac{1}{\left|E_{n, a}\right|^{2}} \geq \frac{1}{K_{n}(\xi, \xi)} \int_{-b}^{b}\left|\int_{-r}^{r} h(s) f(t, s) d s+o(1)\right|^{2} \frac{d t}{\left|E_{n, a}\left(\xi+\frac{t}{\tilde{K}_{n}}\right)\right|^{2} \mu^{\prime}(\xi)} .
$$

From these and (2.7) and (2.9),

$$
\int_{-b}^{b}\left|\int_{-r}^{r} h(s) f(t, s) d s\right|^{2} \frac{d t}{\left|E_{\alpha}(t)\right|^{2}} \leq \int_{-r}^{r} h^{2} .
$$

Now let $r \rightarrow \infty$ and then $b \rightarrow \infty$.

(III) We use Bessel's Inequality in the form

$$
\int S_{n}^{*}\left[g_{r}\right]^{2} \frac{1}{\left|E_{n, a}\right|^{2}} \leq \int g_{r}^{2} \frac{1}{\left|E_{n, a}\right|^{2}}
$$


Here by (2.7) and (2.12),

$$
\int S_{n}^{*}\left[g_{r}\right]^{2} \frac{1}{\left|E_{n, a}\right|^{2}} \geq \frac{1}{K_{n}(\xi, \xi)} \int_{-b}^{b}\left|\int_{-r}^{r} h(s) f(t, s) \frac{d s}{\left|E_{\alpha}(s)\right|^{2}}+o(1)\right|^{2} \frac{d t}{\left|E_{n, a}\right|^{2}\left(\xi+\frac{t}{\kappa_{n}}\right) \mu^{\prime}(\xi)} .
$$

From these last two inequalities and (2.10), we deduce that

$$
\int_{-b}^{b}\left|\int_{-r}^{r} h(s) f(t, s) \frac{d s}{\left|E_{\alpha}(s)\right|^{2}}\right|^{2} \frac{d t}{\left|E_{\alpha}(t)\right|^{2}} \leq \int_{-r}^{r} h^{2}(s) \frac{d s}{\left|E_{\alpha}(s)\right|^{2}} .
$$

Now let $r \rightarrow \infty$ and then $b \rightarrow \infty$.

(IV) We use

$$
\int S_{n}^{*}\left[g_{r}\right]^{2} d \mu=\int S_{n}^{*}\left[g_{r}\right]^{2} \frac{1}{\left|E_{n, a}\right|^{2}} \leq \int g_{r}^{2} \frac{1}{\left|E_{n, a}\right|^{2}} .
$$

Here by (2.12),

$$
\int S_{n}^{*}\left[g_{r}\right]^{2} d \mu \geq \frac{1}{K_{n}(\xi, \xi)} \int_{-b}^{b}\left|\int_{-r}^{r} h(s) f(u, s) \frac{d s}{\left|E_{\alpha}(s)\right|^{2}}+o(1)\right|^{2} \frac{\mu^{\prime}\left(\xi+\frac{t}{\kappa_{n}}\right)}{\mu^{\prime}(\xi)} d t .
$$

From this, (1.15), and (2.10),

$$
\int_{-b}^{b}\left|\int_{-r}^{r} h(s) f(t, s) \frac{d s}{\left|E_{\alpha}(s)\right|^{2}}\right|^{2} d t \leq \int_{-r}^{r} h^{2}(s) \frac{d s}{\left|E_{\alpha}(s)\right|^{2}}
$$

Now let $r \rightarrow \infty$ and then $b \rightarrow \infty$.

\section{Corollary 2.2}

Let $\beta \in[0,2]$ and $h \in L_{2}(\mathbb{R})$.

(a)

$$
\int_{-\infty}^{\infty} \frac{\mathcal{L}[h]^{2}}{\left|E_{\alpha}\right|^{\beta}} \leq \int_{-\infty}^{\infty} h^{2}
$$

(b)

$$
\int_{-\infty}^{\infty} \frac{\mathcal{L}\left[h /\left|E_{\alpha}\right|^{2}\right]^{2}}{\left|E_{\alpha}\right|^{\beta}} \leq \int_{-\infty}^{\infty} \frac{h^{2}}{\left|E_{\alpha}\right|^{2}}
$$

\section{Proof}

(a) The cases $\beta=0,2$ are (I), (II) of Theorem 1.3. So assume $0<\beta<2$, and let $p=\frac{2}{\beta}$, and $q=\frac{p}{p-1}$. We use Hölder's inequality and (I) and (II) of 
Theorem 1.3:

$$
\begin{aligned}
\int_{-\infty}^{\infty} \frac{L[h]^{2}}{\left|E_{\alpha}\right|^{\beta}} & \leq\left(\int_{-\infty}^{\infty} \frac{L[h]^{2}}{\left|E_{\alpha}\right|^{\beta p}}\right)^{1 / p}\left(\int_{-\infty}^{\infty} L[h]^{2}\right)^{1 / q} \\
& =\left(\int_{-\infty}^{\infty} \frac{L[h]^{2}}{\left|E_{\alpha}\right|^{2}}\right)^{1 / p}\left(\int_{-\infty}^{\infty} L[h]^{2}\right)^{1 / q} \\
& \leq\left(\int_{-\infty}^{\infty} h^{2}\right)^{1 / p+1 / q}
\end{aligned}
$$

by the cases $\beta=0,2$.

(b) We use Hölder's inequality and (III) and (IV) of Theorem 1.3 as in (a).

\section{Proof of Corollary 1.4}

Recall from Theorem 1.2 that $f$ is the reproducing kernel for $\mathcal{H}\left(E_{\alpha}\right)$. Then if $g \in P W_{\sigma}$,

$$
L\left[\frac{g}{\left|E_{\alpha}\right|^{2}}\right](x)=\int_{-\infty}^{\infty} g(s) f(s, x) \frac{d s}{\left|E_{\alpha}(s)\right|^{2}}=g(x) .
$$

Then (b) of Corollary 2.2 gives for such $g$, and $\beta \in[0,2]$,

$$
\int_{-\infty}^{\infty} \frac{|g|^{2}}{\left|E_{\alpha}\right|^{\beta}} \leq \int_{-\infty}^{\infty} \frac{|g|^{2}}{\left|E_{\alpha}\right|^{2}}
$$

By Hőlder's inequality, with $p>1, q=\frac{p}{p-1}$, and weight $\frac{|g|^{2}}{\left|E_{\alpha}\right|^{\beta}}$,

$$
\begin{aligned}
\int_{-\infty}^{\infty} \frac{|g|^{2}}{\left|E_{\alpha}\right|^{\beta}} & \leq \int_{-\infty}^{\infty} \frac{|g|^{2}}{\left|E_{\alpha}\right|^{\beta}\left|E_{\alpha}\right|^{2-\beta}} \\
& \leq\left(\int_{-\infty}^{\infty} \frac{|g|^{2}}{\left|E_{\alpha}\right|^{\beta}} \frac{1}{\left|E_{\alpha}\right|^{(2-\beta) p}}\right)^{\frac{1}{p}}\left(\int_{-\infty}^{\infty} \frac{|g|^{2}}{\left|E_{\alpha}\right|^{\beta}}\right)^{1 / q}
\end{aligned}
$$

and cancelling the powers of $\int_{-\infty}^{\infty} \frac{|g|^{2}}{\left|E_{\alpha}\right|^{\beta}}$, gives

$$
\left(\int_{-\infty}^{\infty} \frac{|g|^{2}}{\left|E_{\alpha}\right|^{\beta}}\right)^{1 / p} \leq\left(\int_{-\infty}^{\infty} \frac{|g|^{2}}{\left|E_{\alpha}\right|^{\beta+(2-\beta) p}}\right)^{\frac{1}{p}},
$$

for any $p>1$. Since $\gamma=\beta+(2-\beta) p$ may assume any value in $(2, \infty)$, we obtain (1.21).

We shall need the Gauss type quadrature formula, with nodes $\left\{t_{j n}\right\}$ including the point $\xi$ :

$$
\sum_{j} \lambda_{n}\left(t_{j n}\right) P\left(t_{j n}\right)=\int P(t) d \mu(t),
$$


for all polynomials $P$ of degree $\leq 2 n-2 \quad$ [7, p. 21, Theorem 2.2]. The $\left\{t_{j n}\right\}$ are the zeros of $L_{n}(t, \xi)=(t-\xi) K_{n}(t, \xi)$, and moreover, if $j \neq k$, $K_{n}\left(t_{j n}, t_{k n}\right)=0$. Recall too that $\lambda_{n}$ is the $n$th Christoffel function for $\mu$,

$$
\lambda_{n}(x)=\frac{1}{K_{n}(x, x)} .
$$

Let us order the nodes as

$$
\ldots<t_{-2, n}<t_{-1, n}<t_{0, n}=\xi<t_{1, n}<t_{2, n}<\ldots<
$$

and write

$$
t_{j n}=\xi_{n}+\frac{\rho_{j n}}{\kappa_{n}} \Leftrightarrow \rho_{j n}=\kappa_{n}\left(t_{j n}-\xi\right)
$$

\section{Lemma 2.3}

(a) For each fixed $j$, as $n \rightarrow \infty$ through $\mathcal{S}$,

$$
\rho_{j n} \rightarrow \rho_{j},
$$

where $\rho_{0}=0$ and

$$
\cdots \leq \rho_{-2} \leq \rho_{-1}<0<\rho_{1} \leq \rho_{2} \leq \ldots .
$$

(b) The function $f(0, z)$ has simple zeros at $\rho_{j}, j \neq 0$, and no other zeros.

(c) Let $\ell \geq 1,\left\{c_{j}\right\}_{j=-\ell}^{\ell} \subset \mathbb{R}$, and

$$
P_{n}(t)=\sum_{j=-\ell}^{\ell} c_{j} \frac{K_{n}\left(t, t_{j n}\right)}{K_{n}\left(t_{j n}, t_{j n}\right)}
$$

Then as $n \rightarrow \infty$ through $\mathcal{S}$,

$$
P_{n}\left(\xi+\frac{a}{\kappa_{n}}\right) \rightarrow \sum_{j=-\ell}^{\ell} c_{j} \frac{f\left(a, \rho_{j}\right)}{f\left(\rho_{j}, \rho_{j}\right)},
$$

uniformly for a in compact subsets of $\mathbb{C}$, and

$$
K_{n}(\xi, \xi) \int P_{n}^{2} d \mu \rightarrow \sum_{j=-\ell}^{\ell} \frac{c_{j}^{2}}{f\left(\rho_{j}, \rho_{j}\right)} .
$$

\section{Proof}

(a) (b) These are part of [12, Theorem 5.3(a), Theorem 5.4], and follow easily from the uniform convergence of $f_{n}$ to $f$, and Hurwitz' Theorem.

(c) We see that for fixed $j$, as $n \rightarrow \infty$ through $\mathcal{S}$,

$$
\begin{aligned}
& \frac{K_{n}\left(\xi+\frac{a}{\kappa_{n}}, t_{j n}\right)}{K_{n}\left(t_{j n}, t_{j n}\right)} \\
= & \frac{f_{n}\left(a, \rho_{j n}\right)}{f_{n}\left(\rho_{j n}, \rho_{j n}\right)} \rightarrow \frac{f\left(a, \rho_{j}\right)}{f\left(\rho_{j}, \rho_{j}\right)} .
\end{aligned}
$$


Then (2.18) follows. Next,

$$
\begin{aligned}
& \int P_{n}^{2} d \mu \\
= & \sum_{|j|,|k| \leq \ell} c_{j} c_{k} \int \frac{K_{n}\left(t, t_{j n}\right)}{K_{n}\left(t_{j n}, t_{j n}\right)} \frac{K_{n}\left(t, t_{k n}\right)}{K_{n}\left(t_{k n}, t_{k n}\right)} d \mu(t) \\
= & \sum_{j=-\ell}^{\ell} c_{j}^{2} \frac{1}{K_{n}\left(t_{j n}, t_{j n}\right)}=\frac{1}{K_{n}(\xi, \xi)} \sum_{j=-\ell}^{\ell} c_{j}^{2} \frac{1}{f_{n}\left(\rho_{j n}, \rho_{j n}\right)}
\end{aligned}
$$

by the reproducing kernel property, and since $K_{n}\left(t_{j n}, t_{k n}\right)=0$ for $j \neq k$.

Then (2.19) follows.

\section{Proof of Theorem 1.5}

(a) Let $b>0$. With $g_{r}$ defined by (2.8), we have from (2.7) and (2.12),

$$
\begin{aligned}
& \int_{\xi-\frac{b}{\bar{K}_{n}}}^{\xi+\frac{b}{\bar{K}_{n}}}\left(g_{r}-S_{n}^{*}\left[g_{r}\right]\right)^{2} \frac{1}{\left|E_{n, a}\right|^{2}} \\
= & \frac{1}{K_{n}(\xi, \xi)}\left[\int_{-b}^{b}\left(h(u)-\int_{-r}^{r} h(s) f(u, s) \frac{d s}{\left|E_{\alpha}(s)\right|^{2}}\right)^{2} \frac{d u}{\left|E_{\alpha}(u)\right|^{2}}+o(1)\right] .
\end{aligned}
$$

Moreover, if $P_{n}$ is given by (2.17), then (2.7), (2.18), (2.19) give

$$
\begin{aligned}
& \int_{-\infty}^{\infty}\left(g_{r}-P_{n}\right)^{2} \frac{1}{\left|E_{n, a}\right|^{2}} \\
& =\int_{\xi-\frac{r}{\tilde{K}_{n}}}^{\xi+\frac{r}{\bar{K}_{n}}} g_{r}^{2} \frac{1}{\left|E_{n, a}\right|^{2}}-2 \sum_{|j| \leq \ell} c_{j} \int_{\xi-\frac{r}{\tilde{K}_{n}}}^{\xi+\frac{r}{\bar{K}_{n}}} g_{r}(t) \frac{K_{n}\left(t, t_{j n}\right)}{K_{n}\left(t_{j n}, t_{j n}\right)} \frac{d t}{\left|E_{n, a}(t)\right|^{2}}+\int_{-\infty}^{\infty} P_{n}^{2} d \mu \\
& =\frac{1}{K_{n}(\xi, \xi)}\left[\int_{-r}^{r} \frac{h^{2}}{\left|E_{a}\right|^{2}}-2 \sum_{|j| \leq \ell} c_{j} \int_{-r}^{r} h(t) \frac{f\left(t, \rho_{j}\right)}{f\left(\rho_{j}, \rho_{j}\right)} \frac{d t}{\left|E_{a}(t)\right|^{2}}+\sum_{|j| \leq \ell} \frac{c_{j}^{2}}{f\left(\rho_{j}, \rho_{j}\right)}+o(1)\right] \\
& =\frac{1}{K_{n}(\xi, \xi)}\left[\begin{array}{c}
\int_{-r}^{r}\left(h(t)-\sum_{|j| \leq \ell} c_{j} \frac{f\left(t, \rho_{j}\right)}{f\left(\rho_{j}, \rho_{j}\right)}\right)^{2} \frac{d t}{\left|E_{a}(t)\right|^{2}} \\
-\int_{-r}^{r}\left(\sum_{|j| \leq \ell} c_{j} \frac{f\left(t, \rho_{j}\right)}{f\left(\rho_{j}, \rho_{j}\right)}\right)^{2} \frac{d t}{\left|E_{a}(t)\right|^{2}}+\sum_{|j| \leq \ell} \frac{c_{j}^{2}}{f\left(\rho_{j}, \rho_{j}\right)}+o(1)
\end{array}\right] .
\end{aligned}
$$

Combining the above relations, with the best approximation property of partial sums of orthonormal expansions, namely,

$$
\int\left(g_{r}-S_{n}^{*}\left[g_{r}\right]\right)^{2} \frac{1}{\left|E_{n, a}\right|^{2}} \leq \int\left(g_{r}-P_{n}\right)^{2} \frac{1}{\left|E_{n, a}\right|^{2}}
$$


we obtain

$$
\begin{aligned}
& \int_{-b}^{b}\left(h(u)-\int_{-r}^{r} h(s) f(u, s) \frac{d s}{\left|E_{\alpha}(s)\right|^{2}}\right)^{2} \frac{d u}{\left|E_{\alpha}(u)\right|^{2}} \\
\leq & \int_{-r}^{r}\left(h(u)-\sum_{|j| \leq \ell} c_{j} \frac{f\left(u, \rho_{j}\right)}{f\left(\rho_{j}, \rho_{j}\right)}\right)^{2} \frac{d u}{\left|E_{a}(u)\right|^{2}} \\
& -\int_{-r}^{r}\left(\sum_{|j| \leq \ell} c_{j} \frac{f\left(u, \rho_{j}\right)}{f\left(\rho_{j}, \rho_{j}\right)}\right)^{2} \frac{d u}{\left|E_{a}(u)\right|^{2}}+\sum_{|j| \leq \ell} \frac{c_{j}^{2}}{f\left(\rho_{j}, \rho_{j}\right)} .
\end{aligned}
$$

Let $r \rightarrow \infty$ and use the orthonormality of $\left\{\frac{f\left(\cdot, \rho_{j}\right)}{\sqrt{f\left(\rho_{j}, \rho_{j}\right)}}\right\}$, recall (1.22). We obtain

$$
\begin{aligned}
& \int_{-b}^{b}\left(h(u)-\mathcal{L}\left[\frac{h}{\left|E_{\alpha}\right|^{2}}\right](u)\right)^{2} \frac{d u}{\left|E_{\alpha}(u)\right|^{2}} \\
\leq & \int_{-\infty}^{\infty}\left(h(u)-\sum_{|j| \leq \ell} c_{j} \frac{f\left(u, \rho_{j}\right)}{f\left(\rho_{j}, \rho_{j}\right)}\right)^{2} \frac{d u}{\left|E_{a}(u)\right|^{2}} .
\end{aligned}
$$

The limits are justified as $h(\cdot), f\left(\cdot, \rho_{j}\right) \in L_{2}(\mathbb{R})$. We now let $b \rightarrow \infty$ and then $\ell \rightarrow \infty$, and recall from (1.23) that every $g \in P W_{\sigma}$ admits an orthonormal expansion in terms of $\left\{\frac{f\left(\cdot, \rho_{j}\right)}{\sqrt{f\left(\rho_{j}, \rho_{j}\right)}}\right\}$, with $c_{j}=g\left(\rho_{j}\right)$ for all $j$.

We then obtain

$$
\begin{aligned}
& \int_{-\infty}^{\infty}\left(h(u)-\mathcal{L}\left[\frac{h}{\left|E_{\alpha}\right|^{2}}\right](u)\right)^{2} \frac{d u}{\left|E_{\alpha}(u)\right|^{2}} \\
\leq & \int_{-\infty}^{\infty}(h-g)^{2}(u) \frac{d u}{\left|E_{a}(u)\right|^{2}} .
\end{aligned}
$$

Now take inf's over $g$ to get the result.

(b) Let $b>0$. With $g_{r}$ defined by (2.8), we have from (2.11),

$$
\begin{aligned}
& \int_{\xi-\frac{b}{K_{n}}}^{\xi+\frac{b}{K_{n}}}\left(g_{r}-S_{n}\left[g_{r}\right]\right)^{2} d \mu \\
= & \frac{1}{K_{n}(\xi, \xi)}\left[\int_{-b}^{b}\left(h(u)-\int_{-r}^{r} h(s) f(u, s) d s\right)^{2} d u+o(1)\right],
\end{aligned}
$$


while if $P_{n}$ is given by (2.17), we see that

$$
\begin{aligned}
& \int_{-\infty}^{\infty}\left(g_{r}-P_{n}\right)^{2} d \mu \\
= & \int_{\xi-\frac{r}{\bar{K}_{n}}}^{\xi+\frac{r}{\bar{K}_{n}}} g_{r}^{2} d \mu-2 \sum_{|j| \leq \ell} c_{j} \int_{\xi-\frac{r}{\bar{K}_{n}}}^{\xi+\frac{r}{\bar{K}_{n}}} g_{r}(t) \frac{K_{n}\left(t, t_{j n}\right)}{K_{n}\left(t_{j n}, t_{j n}\right)} d \mu(t)+\int_{-\infty}^{\infty} P_{n}^{2} d \mu \\
= & \frac{1}{K_{n}(\xi, \xi)}\left[\int_{-r}^{r} h^{2}-2 \sum_{|j| \leq \ell} c_{j} \int_{-r}^{r} h(u) \frac{f\left(u, \rho_{j}\right)}{f\left(\rho_{j}, \rho_{j}\right)} d u+\sum_{|j| \leq \ell} \frac{c_{j}^{2}}{f\left(\rho_{j}, \rho_{j}\right)}+o(1)\right] .
\end{aligned}
$$

Combining the above relations, and the best approximation/ least squares inequality

$$
\int\left(g_{r}-S_{n}\left[g_{r}\right]\right)^{2} d \mu \leq \int\left(g_{r}-P_{n}\right)^{2} d \mu
$$

we obtain

$$
\begin{aligned}
& \int_{-b}^{b}\left(h(u)-\int_{-r}^{r} h(s) f(u, s) d s\right)^{2} d u \\
\leq & \int_{-r}^{r} h^{2}-2 \sum_{|j| \leq \ell} \frac{c_{j}}{f\left(\rho_{j}, \rho_{j}\right)} \int_{-r}^{r} h(u) f\left(u, \rho_{j}\right) d u+\sum_{|j| \leq \ell} \frac{c_{j}^{2}}{f\left(\rho_{j}, \rho_{j}\right)} .
\end{aligned}
$$

We now let $r \rightarrow \infty$, to deduce that

$$
\begin{aligned}
& \int_{-b}^{b}(h(u)-\mathcal{L}[h](u))^{2} d u \\
\leq & \int_{-\infty}^{\infty} h^{2}-2 \sum_{|j| \leq \ell} \frac{c_{j}}{f\left(\rho_{j}, \rho_{j}\right)} \mathcal{L}[h]\left(\rho_{j}\right)+\sum_{|j| \leq \ell} \frac{c_{j}^{2}}{f\left(\rho_{j}, \rho_{j}\right)} .
\end{aligned}
$$

Next, we let $\ell \rightarrow \infty$ and finally $b \rightarrow \infty$. We obtain

$$
\begin{aligned}
& \int_{-\infty}^{\infty}(h(u)-\mathcal{L}[h](u))^{2} d u \\
\leq & \int_{-\infty}^{\infty} h^{2}-2 \sum_{j=-\infty}^{\infty} \frac{c_{j}}{f\left(\rho_{j}, \rho_{j}\right)} \mathcal{L}[h]\left(\rho_{j}\right)+\sum_{j=-\infty}^{\infty} \frac{c_{j}^{2}}{f\left(\rho_{j}, \rho_{j}\right)} \\
= & \int_{-\infty}^{\infty} h^{2}-\sum_{j=-\infty}^{\infty} \frac{\mathcal{L}[h]\left(\rho_{j}\right)^{2}}{f\left(\rho_{j}, \rho_{j}\right)}+\sum_{j=-\infty}^{\infty} \frac{\left(c_{j}-\mathcal{L}[h]\left(\rho_{j}\right)\right)^{2}}{f\left(\rho_{j}, \rho_{j}\right)} .
\end{aligned}
$$


Now let $g \in P W_{\sigma}$, for simplicity real valued on the real line, and recall the expansion (1.23). Choosing $c_{j}=g\left(\rho_{j}\right)$, we see that

$$
\begin{aligned}
& \int_{-\infty}^{\infty} h^{2}-2 \sum_{j=-\infty}^{\infty} \frac{c_{j}}{f\left(\rho_{j}, \rho_{j}\right)} \mathcal{L}[h]\left(\rho_{j}\right)+\sum_{j=-\infty}^{\infty} \frac{c_{j}^{2}}{f\left(\rho_{j}, \rho_{j}\right)} \\
= & \int_{-\infty}^{\infty} h^{2}-2 \int_{-\infty}^{\infty} h(t) \sum_{j=-\infty}^{\infty} \frac{g\left(\rho_{j}\right)}{f\left(\rho_{j}, \rho_{j}\right)} f\left(\rho_{j}, t\right) d t+\sum_{j=-\infty}^{\infty} \frac{g\left(\rho_{j}\right)^{2}}{f\left(\rho_{j}, \rho_{j}\right)} \\
= & \int_{-\infty}^{\infty} h^{2}-2 \int_{-\infty}^{\infty} h g+\int_{-\infty}^{\infty} \frac{g^{2}}{\left|E_{\alpha}\right|^{2}},
\end{aligned}
$$

by (1.24). The interchange of series and integral is easily justified using (1.23) and (1.24). We continue this as

$$
=\int_{-\infty}^{\infty}(h-g)^{2}+\int_{-\infty}^{\infty}|g|^{2}\left(\frac{1}{\left|E_{\alpha}\right|^{2}}-1\right) .
$$

Taking the inf over all $g \in P W_{\sigma}$, and substituting into the second inequality in (2.20) gives the first inequality in (1.26). Finally, the minimum on the last right-hand side in $(2.20)$ is achieved when $c_{j}=L[h]\left(\rho_{j}\right)$ for all $j$. This gives the second inequality in (1.26).

\section{Proof of Corollary 1.6}

$(\mathrm{I}) \Longleftrightarrow(\mathrm{II})$

The forward implication is immediate. We must prove (II) $\Rightarrow$ (I). We may assume that $g$ is real on the real line. From our hypothesis and (1.26), we obtain

$$
\sum_{j=1}^{\infty} \frac{g^{2}\left(\rho_{j}\right)}{f\left(\rho_{j}, \rho_{j}\right)}=\sum_{j=1}^{\infty} \frac{L[g]^{2}\left(\rho_{j}\right)}{f\left(\rho_{j}, \rho_{j}\right)} \leq \int_{-\infty}^{\infty} g^{2} .
$$

But we already know from (1.24) and (1.21) that

$$
\sum_{j=1}^{\infty} \frac{g^{2}\left(\rho_{j}\right)}{f\left(\rho_{j}, \rho_{j}\right)}=\int_{-\infty}^{\infty} \frac{g^{2}}{\left|E_{a}\right|^{2}} \geq \int_{-\infty}^{\infty} g^{2}
$$

We then obtain

$$
\sum_{j=1}^{\infty} \frac{g^{2}\left(\rho_{j}\right)}{f\left(\rho_{j}, \rho_{j}\right)}=\sum_{j=1}^{\infty} \frac{\mathcal{L}[g]^{2}\left(\rho_{j}\right)}{f\left(\rho_{j}, \rho_{j}\right)}=\int_{-\infty}^{\infty} g^{2}=\int_{-\infty}^{\infty} \frac{g^{2}}{\left|E_{a}\right|^{2}}
$$

Then (1.26) gives

$$
\int_{-\infty}^{\infty}(g-\mathcal{L}[g])^{2}=0
$$

so $g \equiv \mathcal{L}[g]$.

(II) $\Longleftrightarrow($ III $)$

If (II) holds, then (2.21) gives (1.29). Conversely if (1.29) holds, then choosing the 1 st inequality in $(1.26)$ gives $g \equiv \mathcal{L}[g]$ and hence both $(1.27)$ and 
(1.28).

Finally, if any of (I), (II), or (III) hold, then we can use $g=\mathcal{L}[g]$ for all $g$ in $P W_{\sigma}$ to show that $f$ is the sinc kernel. Indeed, let us apply this, for a given $t$, to

$$
g_{t}(s)=\frac{\sin \sigma(s-t)}{\pi(s-t)},
$$

and use [23, Cor. 1.10 .5 , p. 95$]$ that this is the reproducing kernel for $P W_{\sigma}$. We obtain

$$
g_{t}(x)=\mathcal{L}\left[g_{t}\right](x)=f(x, t),
$$

so that $f$ is the sinc kernel. In the other direction, if $f$ is the sinc kernel, we immediately have $g=\mathcal{L}[g]$ for all $g$ in $P W_{\sigma}$.

\section{REFERENCES}

[1] A. Avila, J. Last, and B. Simon, Bulk universality and clock spacing of zeros for ergodic Jacobi matrices with a.c. spectrum, submitted.

[2] J. Baik, T. Kriecherbauer, K. T-R. McLaughlin, P.D. Miller, Uniform Asymptotics for Polynomials Orthogonal with respect to a General Class of Discrete Weights and Universality Results for Associated Ensembles, Princeton Annals of Mathematics Studies, 2006.

[3] L. de Branges, Hilbert Spaces of Entire Functions, Prentice Hall, New Jersey, 1968.

[4] P. Deift, Orthogonal Polynomials and Random Matrices: A Riemann-Hilbert Approach, Courant Institute Lecture Notes, Vol. 3, New York University Pres, New York, 1999.

[5] M. Findley, Universality for Regular Measures satisfying Szeg"o's Condition, J. Approx. Theory, 155(2008), 136-154.

[6] P. J. Forrester, Log-gases and Random matrices, online book, http://www.ms.unimelb.edu.au/ ${ }^{\sim}$ matpjf/matpjf.html .

[7] G. Freud, Orthogonal Polynomials, Pergamon Press/ Akademiai Kiado, Budapest, 1971.

[8] A.B. Kuijlaars and M. Vanlessen, Universality for Eigenvalue Correlations from the Modified Jacobi Unitary Ensemble, International Maths. Research Notices, 30(2002), 1575-1600.

[9] Eli Levin and D.S. Lubinsky, Universality Limits in the Bulk for Varying Measures, Advances in Mathematics, 219(2008), 743-779.

[10] D.S. Lubinsky, A New Approach to Universality Limits involving Orthogonal Polynomials, Annals of Mathematics, 170(2009), 915-939.

[11] D.S. Lubinsky, Universality Limits in the Bulk for Arbitrary Measures on Compact Sets, J. d'Analyse de Math., 106(2008), 373-394.

[12] D.S. Lubinsky, Universality Limits for Random Matrices and de Branges Spaces of Entire Functions, Journal of Functional Analysis, 256(2009), 3688-3729.

[13] D.S. Lubinsky, Applications of New Geronimus Identities for Real Orthogonal Polynomials, manuscript.

[14] Y.L. Lyubarskii and K. Seip, Weighted Paley-Wiener Spaces, Journal Amer. Math. Soc., 15(2002), 979-1006.

[15] K. T.-R. McLaughlin and P. Miller, The $\bar{\partial}$ steepest descent method and the asymptotic behavior of polynomials orthogonal on the unit circle with fixed and exponentially varying nonanalytic weights, Intern. Math. Res. Papers, 2006, Article ID 48673, pages $1-77$. 
[16] K. T.-R. McLaughlin and P. Miller, The $\bar{\partial}$ steepest descent method for orthogonal polynomials on the real line with varying weights, Int. Math. Res. Not. IMRN 2008, Art. ID rnn 075, 66 pp.

[17] M.L. Mehta, Random Matrices, 2nd edn., Academic Press, Boston, 1991.

[18] A. Moreno, A. Martinez-Finkelshtein, V.L. Sousa, Asymptotics of orthogonal polynomials for a weight with a jump on [-1,1], manuscript.

[19] J. Ortega-Cerda and K. Seip, Fourier Frames, Annals of Math., 155(2002), 789-806.

[20] L. Pastur and M. Schcherbina, Universality of the local Eigenvalue Statistics for a Class of Unitary Invariant Random Matrix Ensembles, J. Statistic Phys., 86(1997), 109-147.

[21] B. Simon, Two Extensions of Lubinsky's Universality Theorem, Journal d'Analyse de Mathematique, 105 (2008), 345-362.

[22] B. Simon, The Christoffel-Darboux Kernel, (in) Perspectives in PDE, Harmonic Analysis and Applications, a volume in honor of V.G. Maz'ya's 70th birthday, Proceedings of Symposia in Pure Mathematics, 79 (2008), 295-335.

[23] F. Stenger, Numerical Methods Based on Sinc and Analytic Functions, Springer, New York, 1993.

[24] V. Totik, Universality and fine zero spacing on general sets, to appear in Arkiv för Matematik.

School of Mathematics, Georgia Institute of Technology, Atlanta, Ga 30332-0160, USA., LUBINSKY@MATH.GATECH.EDU 